\title{
Image Compression Algorithm Based on Morphological Associative Memories
}

\author{
Enrique Guzmán ${ }^{1}$, Oleksiy Pogrebnyak ${ }^{2}$, Cornelio Yáñez ${ }^{2}$, and José A. Moreno ${ }^{1}$ \\ ${ }^{1}$ Universidad Tecnológica de la Mixteca \\ \{eguzman, jamoreno\}@mixteco.utm.mx \\ ${ }^{2}$ Centro de Investigación en Computación del Instituto Politécnico Nacional \\ \{olek, cyanez\}@pollux.cic.ipn.mx
}

\begin{abstract}
A new method for image compression based on Morphological Associative Memories (MAM) is proposed. We used MAM at the transformation stage of image coding, thereby replacing the traditional methods such as Discrete Cosine Transform or Wavelet Transform. After applying the MAM, the informative image data are concentrated in a minimum of values. The next stages of image coding can be obtained by taking advantage of this new representation of the image. The main advantage offered by the MAM with respect to the traditional methods is the speed of processing, whereas the compression rate and the obtained signal to noise ratios compete with the traditional methods.
\end{abstract}

Keywords: Image compression, Morphological Associative Memories, Morphological Hetroassociative Memories min.

\section{Introduction}

Traditional methods in image compression often use the Discrete Cosine Transform (DCT) or the Wavelet Transform at the data transformation stage. Actually, DCT is used in many image compression standards like JPEG, MPEG at image transformation stage [6], [7].

1992 was a vitally important period for the area of image compression. Ronald A. DeVore and his collaborators developed a mathematical theory that enables us to use the Wavelet Transform in image compression in [8]. Daubechies and his collaborators proposed a scheme for image compression by the use of the Wavelet Transform. This decomposition provides sub-images corresponding to different levels of resolution and orientation [9]. Lewis and Knowles propose a scheme for image compression based on the 2-D Wavelet Transform [10]. In this scheme, the image is separated into its spatial elements and spectral coefficients using the 2-D Wavelet Transform.

Various methods for coding of image wavelet coefficients are proposed. The first wavelet image coding algorithm "Embedded Zerotree Wavelet Algorithm" (EZW) was proposed by Shapiro in 1993 [11]. Next, Said and Pearlman in [12] proposed a new and better implementation of the EZW, the SPIHT algorithm (Seth Partitioning in Hierarchical Trees), based on the use of data sets organized in hierarchical trees. A new algorithm for image compression known as EBCOT (Embedded Block Coding 
with Optimized Truncation) was proposed by Taubman in 2000 [13]. The Standard JPEG2000 is based fundamentally on the Discrete Wavelet Transform and Embedded Block Coding with Optimal Truncation (EBCOT) [14].

In this paper, a new method for image compression based on Morphological Associative Memories (MAM) created by Ritter, Sussner and Diaz de León in 1998 [15] is proposed. The operations in MAM are based on the morphological operations, dilation and erosion; in other words, they make use of maximums or minimums of sums [15]. This feature distinguishes them from the Hopfield memories [16], which use sums of products. The MAM have turned out to be an excellent tool for recognizing and recovering patterns, even if these display dilative, erosive or random noise [15], [17], [18].

We used MAM (to be more exactly, morphological heteroassociative memories) at the transformation stage of an image compression system. The main advantage offered by the MAM technique with respect to traditional methods used in this stage is the processing speed meanwhile the compression rate and the signal to noise ratio obtained compete with the traditional methods.

\section{Morphological Associative Memories}

The "Mg" generic associative memory scheme is shown in Figure 1, the input patterns and output patters are represented by $\mathbf{x}$ and $\mathbf{y}$ respectively.

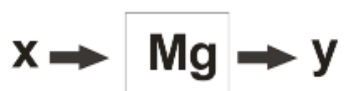

Fig. 1. Associative memory scheme [23]

$$
\mathbf{x}^{T}=\left(x_{1}, x_{2}, \ldots, x_{n}\right) ; \mathbf{y}^{T}=\left(y_{1}, y_{2}, \ldots, y_{n}\right)
$$

$n$ and $m$ are integer positive numbers and represent the dimensions of the input and output patters respectively. Henceforth, let $\left\{\left(\mathbf{x}^{1}, \mathbf{y}^{1}\right),\left(\mathbf{x}^{2}, \mathbf{y}^{2}\right), \ldots,\left(\mathbf{x}^{k}, \mathbf{y}^{k}\right)\right\}$ be $k$ vector pairs defined as the fundamental set of associations [18] that is represented by

$$
\left\{\left(\mathbf{x}^{\mu}, \mathbf{y}^{\mu}\right) \mid \mu=1,2, \ldots, k\right\} .
$$

The Mg associative memory is represented by a matrix and it is generated from the fundamental set of associations. Once the fundamental set is delineated, we define the necessary operations for the learning process and recovery process of a MAM; these operations make use of the maximum $\vee$ and minimum $\wedge$ operators [18], [19].

Let $D$ be a column vector of dimension $m$, and $F$ a row vector of dimension $n$, the maximum product is given by

$$
D \nabla F=C=\left[c_{i j}\right]_{m \times n} .
$$

where $c_{i j}=\left(d_{i}+f_{j}\right)$. Ggeneralizing for a fundamental set of associations: 


$$
c_{i j}=\bigvee_{l=1}^{k}\left(d_{i l}+f_{l j}\right)
$$

The minimum product is given by

$$
D \Delta F=C=\left[c_{i j}\right]_{m \times n}
$$

for a fundamental set of associations $c_{i j}$ is defined by

$$
c_{i j}=\bigwedge_{l=1}^{k}\left(d_{i l}+f_{l j}\right)
$$

On the other hand, let $D=\left[d_{i j}\right]_{m \times n}$ be a matrix and $F=\left[f_{i}\right]_{n}$ a column vector, the calculation of the maximum product $D \nabla F$ gives as result a column vector $C=\left[c_{i}\right]_{m}$, where $c_{i}$ is defined by

$$
c_{i}=\bigvee_{j=1}^{n}\left(d_{i j}+f_{j}\right)
$$

For the minimum product $C=D \Delta F$

$$
c_{i}=\bigwedge_{j=1}^{n}\left(d_{i j}+f_{j}\right)
$$

According to the mode of operation, the associative memories are classified in two groups:

- morphological auto-associative memories (MAAM).

- morphological hetero-associative memories (MHM).

\subsection{Morphological Heteroassociative Memories min}

A MAM is Hetero-associative if $\exists \mu \in\{1,2, \ldots, k\}$ such that $\mathbf{x}^{\mu} \neq \mathbf{y}^{\mu}$. There are two types of MHM: max, symbolized by $\mathbf{M}$, and $\min$, symbolized by W. The MHM min (W) are those that use the maximum product and the minimum operator in their learning phase and the maximum product in their recovery phase.

\section{Learning Phase:}

1. The matrices, $\mathbf{y}^{\mu} \nabla\left(-\mathbf{x}^{\mu}\right)^{t}$, are calculated for each one of the $k$ elements of the fundamental set of associations $\left(\mathbf{x}^{\mu}, \mathbf{y}^{\mu}\right)$.

2. The $\mathbf{W}$ memory is obtained having applied the minimum operator $\wedge$ to the resulting matrices of step $1 . \mathbf{W}$ is given by

$$
\mathbf{W}=\bigwedge_{\mu=\mathbf{1}}^{k}\left[\mathbf{y}^{\mu} \nabla\left(-\mathbf{x}^{\mu}\right)^{t}\right]=\left[w_{i j}\right]_{m \times n}, w_{i j}=\bigwedge_{\mu=1}^{k}\left(y_{i}^{\mu}-x_{j}^{\mu}\right)
$$

\section{Recovery Phase:}

1. The maximum product $\mathbf{W} \nabla \mathbf{x}^{\omega}$ is calculated, where $\omega \in\{1,2, \ldots, k\}$, obtaining a column vector $\mathbf{y}=\left[y_{i}\right]_{m}$, which represent the output patterns associated with $\mathbf{x}^{\omega}$ input patterns, 


$$
\mathbf{y}=\mathbf{W} \nabla \mathbf{x}^{\omega}, y_{i}=\bigvee_{j=1}^{n}\left(w_{i j}+x_{j}^{\omega}\right)
$$

Theorem 2 and Corollary 2.1 of [20] govern the conditions that must be satisfied by MHM min to obtain a perfect recall to output patterns.

Theorem 2: $\mathbf{W} \nabla \mathbf{x}^{\omega}=\mathbf{y}^{\omega} \forall \omega=1, \ldots, k$ if and only if for each $\omega$ and each row index $i=1, \ldots, m$ there are column indexes $j_{i}^{\omega} \in\{1, \ldots, n\}$ such that $m_{i j_{i}^{\omega}}=y_{i}^{\omega}-x_{j_{i}^{\omega}}^{\omega}$ $\forall \omega=1, \ldots, k$. Corollary 2.1: $\mathbf{W} \nabla \mathbf{x}^{\omega}=\mathbf{y}^{\omega} \forall \omega=1, \ldots, k$ if and only if for each row index $i=1, \ldots, m$ and each $\gamma \in\{1, \ldots, k\}$ there is a column index $j_{i}^{\gamma} \in\{1, \ldots, n\}$ such that

$$
\mathbf{x}_{j_{i}^{\gamma}}^{\gamma}=\bigvee_{\varepsilon=1}^{k}\left(x_{j_{i}^{\gamma}}^{\varepsilon}-y_{i}^{\varepsilon}\right)+y_{i}^{\gamma}
$$

On the other hand, Theorem 6 of [20] indicates the amount of noise that is permissible in the input patterns to obtain a perfect recall to output patterns.

Theorem 6: For $\gamma=1, \ldots, k$, let $\tilde{\mathbf{x}}^{\gamma}$ be a corrupted input pattern of $\mathbf{x}^{\gamma}$. Then $\mathbf{W} \nabla \tilde{\mathbf{x}}^{\gamma}=\mathbf{y}^{\gamma}$ if and only if it satisfies that

$$
\tilde{x}_{j}^{\gamma} \leq x_{j}^{\gamma} \vee \bigwedge_{i=1}^{m}\left(\bigvee_{\varepsilon \neq \gamma}\left[y_{i}^{\gamma}-y_{i}^{\varepsilon}+x_{i}^{\varepsilon}\right]\right) \forall j=1, \ldots, n
$$

and for each row index $i \in\{1, \ldots, m\}$ there is a column index $j_{i} \in\{1, \ldots, n\}$ such that:

$$
\tilde{x}_{j i}^{\gamma}=x_{j i}^{\gamma} \vee\left(\bigvee_{\varepsilon \neq \gamma}\left[y_{i}^{\gamma}-y_{i}^{\varepsilon}+x_{j i}^{\varepsilon}\right]\right)
$$

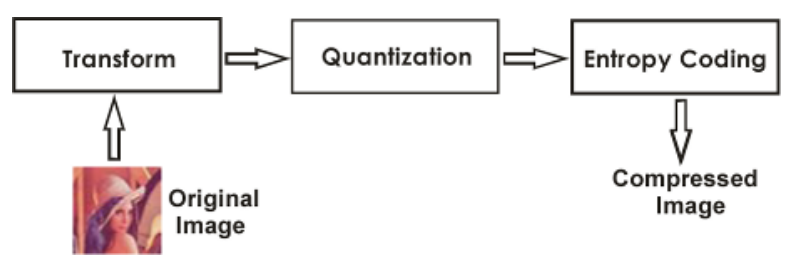

Fig. 2. Scheme of image compression system

\section{Image Transformation Using MHM min}

An image compression system can be composed of three principal stages shown in Figure 2. The transformation stage is the main focus of this study; traditional methods of image compression use the DCT or the Wavelet transform in this stage. We propose the use of the MAM in the transformation stage. MAM satisfies the features of a transformation method: the transformed MAM information is concentrated into a 
minimum of values; the transformed MAM is reversible; the MAM has low memory requirements, uses limited arithmetical precision, and few arithmetical operations.

Let image $\mathbf{A}$ is divided into $\mathbf{N} m^{\prime} \times n^{\prime}$ sub-matrix or image blocks, obtaining $\mathbf{N}=\left(m / m^{\prime}\right) \cdot\left(n / n^{\prime}\right)$ blocks, each of these blocks is divided into $m^{\prime}$ vectors of dimension $n^{\prime}$; these vectors represent the output patterns, $\mathbf{y}^{m^{\prime}}=\left[y_{i}\right]_{n^{\prime}}$, of the MHM min. On the other hand, the input patterns must be known for both image coding and image decoding; besides, the generation of these input patterns is governed by Theorem 2 and the Corollary 2.1 of [20]. $m^{\prime}$ input patterns of dimension $n^{\prime}$ are created

$$
\begin{gathered}
\mathbf{x}^{1}=\left[x_{1}^{1}, x_{2}^{1}, x_{3}^{1}, \ldots, x_{n^{\prime}}^{1}\right] \\
\mathbf{x}^{2}=\left[x_{1}^{2}, x_{2}^{2}, x_{3}^{2}, \ldots, x_{n^{\prime}}^{2}\right] \\
\mathbf{x}^{3}=\left[x_{1}^{3}, x_{2}^{3}, x_{3}^{3}, \ldots, x_{n^{\prime}}^{3}\right] \\
\vdots \\
\mathbf{x}^{m^{\prime}}=\left[x_{1}^{m^{\prime}}, x_{2}^{m^{\prime}}, x_{3}^{m^{\prime}}, \ldots, x_{n^{\prime}}^{m^{\prime}}\right]
\end{gathered}
$$

To satisfy Theorem 2 and the Corollary 2.1, choose the elements of the input patterns with the following conditions:

$$
x_{n^{\prime}}^{m^{\prime}} \begin{cases}=0 & \text { si } m^{\prime} \neq n^{\prime} \\ >a & \text { si } m^{\prime}=n^{\prime} ; \forall a \in \mathbf{A}\end{cases}
$$

Where $a$ is the maximum value that can take an element of the A matrix. Once the input and output patterns are defined, $\mathbf{W}$ is obtained by applying (9) to every submatrix of the image with the set of chosen input patterns shown in Figure 3.
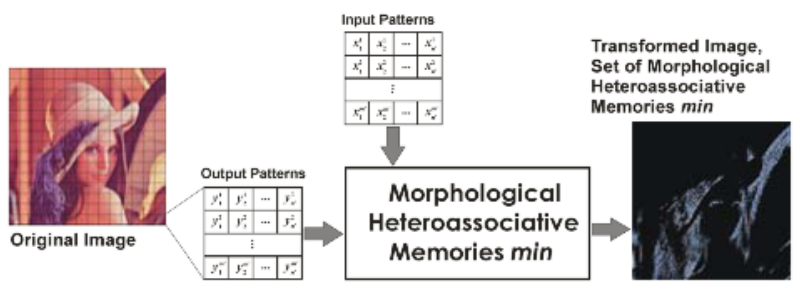

Fig. 3. Scheme of the MHM min applied to an image

As a result of applying a MHM min to an image, $\mathbf{N} m^{\prime} \times n^{\prime}$ associative memories $\mathbf{W}$ are obtained. The image information remains concentrated in a minimum of values. It is possible to take advantage of this new representation of the image in the following stages of image coding.

The next stage of image coding is a uniform scalar quantization. This stage modifies each of the associative memories $\mathbf{W}$. Lets remember that the perfect recall to output patterns is based on the fact that noise appears in the input patterns and not in the associative memories; how, then, does it affect this noise in the associative memory for the recovery of the original output patterns (blocks of the original image)? 
To answer this question, let $\tilde{\mathbf{W}}$ be a corrupted version of the associative memory $\mathbf{W}$ :

$$
\begin{aligned}
\tilde{\mathbf{W}} & =\mathbf{W} \pm r \\
\tilde{w}_{i j} & =w_{i j} \pm r
\end{aligned}
$$

where $r$ represents the noise associated with $\mathbf{W}$.

Considering Theorem 2, its respective Corollary 2.1 and Theorem 3 of [15], we have $\mathbf{y}^{\gamma}=\mathbf{W} \nabla \mathbf{x}^{\gamma}$, bearing in mind the corrupted version of the associative memory, then $\mathbf{y}^{\gamma}=\tilde{\mathbf{W}} \nabla \mathbf{x}^{\gamma}$.

The equation (17) shows that the noise $r$ associated with the associative memory directly affects the output patterns and the perfect recovery of the image.

$$
\begin{aligned}
& \left(\mathbf{W} \nabla \mathbf{x}^{\gamma}\right)_{i}=\underbrace{n}_{j=1}\left(\tilde{w}_{i j}+x_{j}^{\gamma}\right) \geq \tilde{w}_{i j_{i}}+x_{j}^{\gamma}=\tilde{w}_{i j_{i}}+\left(\bigvee_{\varepsilon=1}^{k}\left(x_{j_{i}}^{\varepsilon}-y_{i}^{\varepsilon}\right)+y_{i}^{\gamma}\right) \\
& =\tilde{w}_{i j_{i}}+y_{i}^{\gamma}-\bigwedge_{\varepsilon=1}^{k}\left(y_{i}^{\varepsilon}-x_{j_{i}}^{\varepsilon}\right)=\tilde{w}_{i j_{i}}+y_{i}^{\gamma}-w_{i j_{i}}=w_{i j_{i}} \pm r+y_{i}^{\gamma}-w_{i j_{i}}=y_{i}^{\gamma} \pm r
\end{aligned}
$$

The noise $r$ is associated with the set of associative memories and depends directly on the quantization factor $Q$ used. As a uniform scalar quantization is used, then each of the associative memories is modified as $\tilde{\mathbf{W}}=\mathbf{W} / Q$.

This process is realized using $\mathrm{R}$ numbers to facilitate the coding process, $\tilde{\mathbf{W}}$ is truncated to $\mathrm{Z}$ numbers by

$$
\tilde{\mathbf{W}}=\left\{\begin{array}{cll}
\tilde{\mathbf{W}}-1 & \text { if } & \tilde{\mathbf{W}}^{\mathbf{R}}-\tilde{\mathbf{W}}^{\mathbf{Z}} \leq-0.5 \\
\tilde{\mathbf{W}} & \text { if } & \tilde{\mathbf{W}}^{\mathbf{R}}-\tilde{\mathbf{W}}^{\mathbf{Z}}>-0.5
\end{array},\right.
$$

where $\tilde{\mathbf{W}}^{\mathbf{R}} \in \mathbf{R}, \tilde{\mathbf{W}}^{\mathbf{Z}} \in \mathrm{Z}$ is the integer part of $\tilde{\mathbf{W}}^{\mathbf{R}}$. The $\tilde{\mathbf{W}}$ range is [-249,-13], if the recovery value of $\mathbf{W}$ is given for $\tilde{\mathbf{W}} \times Q$, then noise added to associative memory $\mathbf{W}$ is from $0 \%$ to $23 \%$, and this will directly affect the output patterns or sub-blocks of the image.

The range of $\tilde{\mathbf{W}}$ was obtained from three image fields that $\mathrm{Y}, \mathrm{Cb}$ and $\mathrm{Cr}$. The range of noise was obtained from all the levels of quantification, therefore, the highest percentage of noise corresponds to $Q=10$. Due to characteristics of the quantization, the percentage of noise does not affect the entire image, only a very low percentage of elements of the same.

\subsection{Decoding Process}

After the stages of entropy decoding and inverse quantification, the recovery process is applied using the set of associative memories.

Once the image is transformed into a set of associative memories and knowing the input patterns, the image transformation using MHM min is reversible (see Fig. 4).

When dealing with MMH min, the inverse transformation process makes use of the maximum product $\mathbf{W} \nabla \mathbf{x}^{\omega}$, where $\omega \in\{1,2, \ldots, k\}$, the input patterns $\mathbf{x}$ are defined in 
(14) and $\mathbf{W}$ is an associative memory belonging to the set that forms the transformed image. As a result a set of vectors $\mathbf{y}^{m^{\prime}}=\left[y_{i}\right]_{n^{\prime}}$ are obtained that represent a block of the original recovery image. Since the associative memory $\mathbf{W}$ is affected by noise, then $\tilde{\mathbf{W}} \nabla \mathbf{x}^{\gamma}=\tilde{\mathbf{y}}^{\gamma}$.

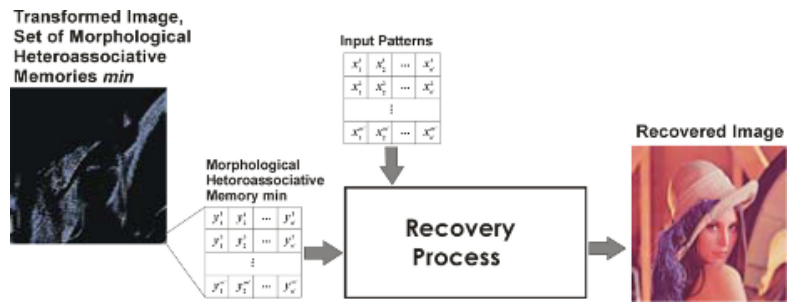

Fig. 4. Recovery algorithm scheme

\section{Results}

The test images are: Lena 512x512, Foreman 352x288 and Akiyo 176x144. The result of applying the MHM min to these images can be seen in Figure 5.

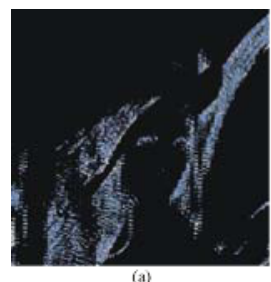

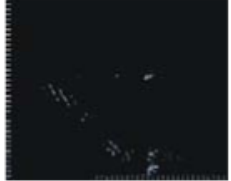

(b)

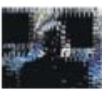

(c)

Fig. 5. Results of applying the MMH $\min$ on $Y$ of: (a) Lena 512x512, (b) Foreman 352x288, (c) Akiyo 176x144

The image transformed by MHM min is quantified using an uniform scalar quantization. Then, standard methods of codification were applied. Such methods include statistical coding, such as arithmetical, Huffman, range and PPM, and dictionary coding, LZ77 and LZP. The results of compression using MHM min on Lena, at different levels of quantification and using diverse codifiers of entropy, is available in Table 1.

From these results one can conclude that the coder which offers best results of compression and signal to noise ratio obtained on the image transformed by a $\mathrm{MMH}$ min is the PPM coding; it is an adaptive statistical method and its operation is based on partial equalization of chains, that is, PPM coding predicts the value of an element based on a sequence of previous elements.

The efficiency of the image coder based on an MAM was compared with results of other image compression methods, JPEG [21], [22], DCT Based Embedded [21], EZW [11], [22], SPIHT [12], [22], EBCOT [13], proving to be competitive with them in the characteristics of compression and signal to noise ratio, Table 2 and Fig. 5. 
Table 1. Comparative results of applying MHM min and several coders on Lena

\begin{tabular}{lcccccc}
\hline & \multicolumn{5}{c}{$\begin{array}{c}\text { Morphological Heteroassociative Memories } \min (\mathbf{8 x 8} \text { block) } \\
\text { Lena (512x512=786486 bytes, 24 bpp) }\end{array}$} \\
\cline { 2 - 7 } & $\mathbf{Q = 1}$ & $\mathbf{Q = 2}$ & $\mathbf{Q = 4}$ & $\mathbf{Q = 6}$ & $\mathbf{Q = 8}$ & $\mathbf{Q = 1 0}$ \\
& PSNR=44.19 & PSNR=42.14 & PSNR=38.12 & PSNR=35.08 & PSNR=32.84 & PSNR=31.02 \\
\hline Range & 108238 bytes & 95971 bytes & 84246 bytes & 77937 bytes & 74124 bytes & 69919 bytes \\
Comp. ratio & $7.27: 1$ & $8.19: 1$ & $9.33: 1$ & $10.09: 1$ & $10.61: 1$ & $11.25: 1$ \\
Bit rate & 3.3 bpp & 2.93 bpp & 2.57 bpp & 2.38 bpp & 2.26 bpp & 2.13 bpp \\
LZ77 & 76903 bytes & 56420 bytes & 37893 bytes & 30201 bytes & 25041 bytes & 20386 bytes \\
Comp. ratio & $10.23: 1$ & $13.94: 1$ & $20.75: 1$ & $26.04: 1$ & $31.41: 1$ & $38.58: 1$ \\
Bit rate & 2.35 bpp & 1.72 bpp & 1.16 bpp & 0.92 bpp & 0.76 bpp & 0.62 bpp \\
LZP & 74727 bytes & 58393 bytes & 40478 bytes & 31505 bytes & 27059 bytes & 20801 bytes \\
Comp. ratio & $10.52: 1$ & $13.47: 1$ & $19.43: 1$ & $24.96: 1$ & $29.06: 1$ & $37.81: 1$ \\
Bit rate & 2.28 bpp & 1.78 bpp & 1.24 bpp & $0.96 \mathrm{Bpp}$ & 0.83 bpp & 0.63 bpp \\
PPM & 46504 bytes & 32858 bytes & 21682 bytes & 16793 bytes & 14205 bytes & 11426 bytes \\
Comp. ratio & $16.91: 1$ & $23.93: 1$ & $36.27: 1$ & $46.83: 1$ & $55.36: 1$ & $68.83: 1$ \\
Bit rate & 1.42 bpp & 1 bpp & 0.66 bpp & 0.51 bpp & 0.43 bpp & 0.35 bpp \\
\hline
\end{tabular}

Table 2. Comparison between MMH min and traditional methods on Lena 512x512

\begin{tabular}{lcc}
\hline \multicolumn{3}{c}{ Lena 512x512 } \\
\hline & Bit Rate & PSNR \\
\hline Bseline JPEG [21], [22] & 0.25 & 31.6 \\
& 0.50 & 34.9 \\
DCT-Based Embedded [21] & 0.25 & 32.25 \\
& 0.50 & 36.0 \\
EZW [11], [22] & 0.25 & 33.17 \\
& 0.50 & 36.28 \\
SPIHT [12], [22] & 0.25 & 34.1 \\
& 0.50 & 37.2 \\
EBCOT [13] & 0.25 & 34.40 \\
& 0.50 & 37.49 \\
MHM min & 0.43 & 32.84 \\
& 0.51 & 35.08 \\
\hline
\end{tabular}

Table 3. Processing speed comparison between MHM min, DCT and DWT on Lena 512x512

\begin{tabular}{lc}
\hline & Number of operations \\
Discrete Cosine Transform & $7,340,032$ sums, $1,310,720$ multiplications \\
Wavelet Transform (three-stage) & $1,032,192$ sums, 344,064 shifts \\
MHM min & 262,144 sums, 262,144 comparisons \\
\hline
\end{tabular}

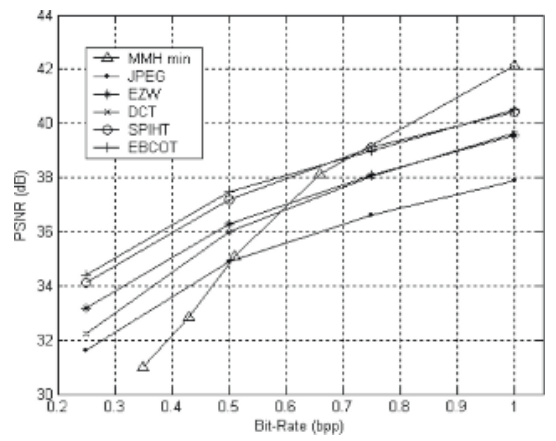

Fig. 6. MMH $\min$ in comparison to traditional methods on Lena $512 \times 512$ 
Morphological associative memories were shown to be a more efficient transformation process in the processing speed with regard to the traditional methods of transformation. Table 3 shows the number of operations the MHM min, the DCT and the WT needed to transform the image Lena $512 \times 512$.

\section{Conclusions}

The use of Morphological Associative Memories in the transformation stage of an image compressor has demonstrated a high competitiveness in its efficiency compared to traditional methods based on DCT or wavelet transform. Furthermore, a MAM has low computational complexity since its calculation is based on operations of comparisons and sums. A better response in the signal to noise ratio can be achieved using associative morphologic bidirectional memories (AMBM) that are robust to random noise.

The quantization process used in this coder is the simplest and thereby offers limited efficiency in compression. Vector quantization is an alternative method that can be used to obtain better results of compression ratio and signal to noise ratio. By basing its functioning on an algorithm of search of equalization, vector quantization makes it a slow process and of considerable computational complexity..

Acknowledgements. This study was supported by the Centro de Investigación en Computación of the Instituto Politécnico Nacional as part of research project CGPI No. 2004285.

\section{References}

1. N. Ahmed, T. Natrajan and K. R. Rao. "Discrete Cosine Transform". IEEE Transactions on Computer, Vol. 23, 1974, pp. 90-93.

2. W. H. Chen, C. H. Smith and S.C Fralick. "A Fast Computational Algorithm for the Discrete Cosine Transform”. IEEE Transactions on Communications, Vol. COM-25, 1977, pp. 1004-1009.

3. Y. Arai, T. Agui y M. Nakajima. "A Fast DCT-SQ Scheme for Image" Transactions of the IEICE, Vol. E 71, No 11, 1988, pp. 1095-1097.

4. B. D. Tseng and W. C. Miller. "On Computing the Discrete Cosine Transform". IEEE Transactions on Computer, C-27, 10, 1978, pp. 966-968.

5. S. Winograd. "On Computing the Discrete Fourier Transform". Proceedings of the National Academy of Sciences of the United States of America, Vol. 73, No. 4, 1976, pp. 1005-1006.

6. G. K. Wallace. "The JPEG Still Picture Compression Standard". Communications of the ACM, Vol. 34, No. 4, 1991, pp. 30-44.

7. ISO, Digital compression and coding of continuous-tone still images: Requirements and guidelines, ISO/IEC IS 10918-1, 1994.

8. R. A. DeVore, B. Jawerth and B. J. Lucier. "Image Compression Through Wavelet Transform Coding". IEEE Transactions on Information Theory, Vol 38, No. 2, 1992, pp 719-746. 
9. M. Antonini, M. Barlaud, P. Mathieu and I. Daubechies. "Image Coding Using Wavelet Transform". IEEE Transactions on Image Processing, Vol. 1, No. 2, 1992, pp. 205-220.

10. A. S. Lewis and G. Knowles. "Image Compression Using the 2-D Wavelet Transform". IEEE Transactions on Image Processing, Vol. 1, No. 2, 1992, pp. 244-250.

11. J. M. Shapiro. "Embedded Image Coding Using Zerotrees of Wavelet Coefficients". IEEE Transactions on Signal Processing, Vol. 41, No. 12, 1993, pp. 3445-3462.

12. A. Said and W. A. Pearlman. "A New, Fast, and Efficient Image Codec Based on Set Partitioning in Hierarchical Trees". IEEE Transactions on Circuits and Systems for Video Technology, Vol. 6, No. 3, 1996, pp. 243-250.

13. D. Taubman. "High Performance Scalable Image Compression with EBCOT". IEEE Transactions on Image Processing, Vol. 9, No. 7, 2000, pp. 1158-1170.

14. Joint Photographic Experts Group. JPEG 2000 Part I Final Committee Draft Version 1.0 ISO/IEC JTC 1/SC 29/WG 1, N1646R, (ITU-T SG8), 2000.

15. G. X. Ritter, P. Sussner and J. L. Díaz de León. "Morphological Associative Memories". IEEE Transactions on Neural Networks, Vol. 9, No. 2, 1998, pp. 281-293.

16. J. J. Hopfield. "Neural Networks and Physical Systems with Emergent Collective Computational Abilities". Proceedings of the National Academy of Sciences of the USA, Vol. 79, 1982, pp. 2554-2558.

17. C. Castellanos, J. L. Díaz De León y A. Sánchez. "Análisis Experimental con las Memorias Asociativas Morfológicas". XXI Congreso Internacional de Ingeniería Electrónica, Electro 99, Instituto Tecnológico de Chihuahua, Chih., México, 1999, pp. 11-16.

18. C. Yáñes y J. L. Díaz de León. Memorias Morfológicas Heteroasociativas. Centro de Investigación en Computación, IPN, México, IT 57, Serie Verde, ISBN 970-18-6697-5, 2001.

19. C. Yáñes y J. L. Díaz de León. Memorias Morfológicas Autoasociativas. Centro de Investigación en Computación, IPN, México, IT 58, Serie Verde, ISBN 970-18-6698-3, 2001.

20. G. X. Ritter, J. L. Díaz de León and P. Sussner. "Morphological Bidirectional Associative Memories". Neural Networks, Vol. 12, No. 6, 1999, pp. 851-867.

21. Z. Xiong, O. G. Guleryuz and M. T. Orchard. "A DCT-Based Embedded Image Coder". IEEE Signal processing Letters, Vol. 3, No. 11, 1996, pp. 289-290.

22. Z. Xiong, K. Ramchandran, M. T. Orchard and Y. Zhang. "A Comparative Study of DCTand Wavelet-Based Image Coding". IEEE Transactions on Circuits and Systems for Video Technology, Vol 9, No 5, 1999, pp. 692-695. 